\title{
Vulnerabilidad-resiliencia ante el proceso de riesgo-desastre: Un análisis desde la ecología política
}

\author{
José Sandoval-Díaz \\ Centro de Estudios Ñuble, Universidad del Bío Bío, Chillán, Chile. \\ Email: jsandoval@ubiobio.cl
}

\begin{abstract}
Resumen: Las nociones de vulnerabilidad y resiliencia se han instalado como categorías explicativas centrales del proceso de riesgo-desastre, tanto en lo académico como institucional. No obstante, ambas nociones se han configurado como significantes flotantes del campo discursivo de la gestión de riesgo de desastres, presentando distintos supuestos, expresiones, énfasis y formas relacionales. A partir de esto el presente trabajo busca analizar, en un primer momento la construcción del discurso de la vulnerabilidad-resiliencia en el campo académico-institucional, para posteriormente, explorar la producción de la vulnerabilidad y la emergencia de la resiliencia local, bajo el prisma del capitaloceno de corte extractivista de Chile. A modo de conclusión afirmamos la importancia de incorporar en la discusión de la reducción de riesgo de desastre, tanto el esclarecimiento conceptual, como la relación dialéctica diferencial entre vulnerabilidad-resiliencia a escala glocal, comprendiendo que el fortalecimiento de capacidades debe ir acompañado de la reducción de las injusticias espaciales y ambientales.
\end{abstract}

Palabras claves: Desastre socionatural; vulnerabilidad social; resiliencia; capitaloceno; ecología política.

\section{Vulnerability-resilience to the risk-disaster process: An analysis from the political ecology}

Abstract: The notions of vulnerability and resilience have been installed as central explanatory categories for the process of disaster risk, both in the academic and institutional spheres. Nevertheless, both notions have been configured as floating signifiers of the discursive field of disaster risk management, presenting different assumptions, expressions, emphasis and relational forms. From this, the present work seeks to analyze, at first, the construction of the discourse of vulnerability-resilience in the academic-institutional field, to later explore the production of vulnerability and the emergence of local resilience, under the prism of Chile's extractivist capitalocene. By way of conclusion we affirm the importance of incorporating into the discussion of disaster risk reduction both conceptual clarification and the differential dialectic relationship between vulnerability and resilience at a global scale, understanding that the strengthening of capacities must be accompanied by the reduction of spatial and environmental injustices.

Keywords: Socionatural disaster; social vulnerability; resilience; capitalocene; political ecology.

\section{Vulnerabilidade-resiliência ao processo de risco-desastre: uma análise desde a ecologia política}

Resumo: As noções de vulnerabilidade e resiliência tem-se instalado como categorias explicativas centrais do processo de risco-desastre, tanto acadêmica quanto institucionalmente. No entanto, ambas as noções foram configuradas como significantes flutuantes do campo discursivo do gerenciamento de riscos de desastres, apresentando diferentes supostos, expressões, ênfases e formas relacionais. A partir disso, o presente trabalho procura analisar, inicialmente, a construção do discurso vulnerabilidade-resiliência no campo acadêmico-institucional e, em seguida, explorar a produção da vulnerabilidade e o surgimento da resiliência local, sob o prisma do capitaloceno de tipo extrativista no Chile. Concluindo, afirmamos a importância de incorporar na discussão sobre redução de riscos de desastres, tanto o esclarecimento conceitual quanto a relação dialética diferencial entre vulnerabilidade-resiliência no nível glocal, entendendo que o fortalecimento de 
capacidades deve ser acompanhado de redução das injustiças espaciais e ambientais.

Palavras-chave: Desastre socionatural; vulnerabilidade social; resiliência; capitaloceno; ecologia política.

$$
* * *
$$

\section{Introducción}

En la actualidad, los desastres socionaturales (acontecimientos destructivos producidos por la interacción entre un peligro natural y la vulnerabilidad estructural), se han convertido en uno de los principales obstáculos para el desarrollo tanto de países, como de medios de vida territoriales (Obradovich, Tingley, y Rahwan, 2018; UN Office for Disaster Risk Reduction [UNDRR], 2015a).

De acuerdo con Pesaresi et al. (2017), a escala global, 2.700 millones de personas viven expuestas a terremotos, alrededor de mil millones a inundaciones y 414 millones viven cerca de los 220 volcanes más peligrosos. Para América Latina y el Caribe (ALC), sólo en los últimos diez años, los desastres han dejado un saldo de más de 45.000 muertos, 62 millones de damnificados y daños materiales que superan los US\$154.000 millones; Con un promedio de 40 desastres importantes por año, ocupando el segundo lugar después de Asia (UNDRR, 2015b).

Estas condiciones geográficas, geológicas, geomorfológicas y meteorológicas, exponen a la región a un amplio repertorio de amenazas naturales, a lo cual debe sumarse un modelo económico neocolonial fundado en la extracción de bienes comunes, el aumento sostenido de pobreza urbana, desigualdades sociales, racismo estructural, opresión basada en el género, degradación ambiental y ausencia de un ordenamiento territorial basado en el riesgo (Alcántara-Ayala, 2019; Atallah, 2016). A esto se suma el cambio ambiental global, el cual incrementaría la presencia de eventos extremos en ciertos periodos y regiones, tanto a nivel de ocurrencia, magnitud y severidad de impacto (Intergovernmental Panel on Climate Change [IPCC], 2014).

Para el caso de las denominadas regiones subdesarrolladas, Wisner, Blaikie, Cannon y Davis (2004) han señalado a la vulnerabilidad social como una de las causas de fondo de las diversas fragilidades emergentes ante el proceso de riesgo-desastre. Para ellos, a diferencia de los enfoques fisicalistas/naturalistas que enfatizan el papel del peligro natural, la centralidad radicaría en la vulnerabilidad social, en tanto condición de base estructural, construida y dinámica configuradora de la susceptibilidad espacial del riesgo (Maskrey, 2011). No obstante, si bien esta perspectiva estructuralista ha relevado el papel causal de los procesos políticos, económicos e históricos (Lavell, 2004), está ha descuidado el papel agentivo de las comunidades expuestas-susceptibles que resisten y/o transforman estas estructuras de vulnerabilización, reduciendo su papel a una mera «representación fetichista de víctimas homogéneas» (Oliver-Smith, Alcántara-Ayala, Burton y Lavell, 2016; Astudillo y Sandoval, 2019).

De acuerdo con esto, en las últimas décadas la literatura se ha centrado en algunas experiencias exitosas de comunidades que se han anticipado y/o recuperado con escasa o nula asistencia externa, siendo categorizadas como resilientes (Tierney, 2014; Manyena, 2006). Aproximadamente, desde fines de los 90 del siglo pasado, la noción de resiliencia se ha instalado en el campo académico-institucional de reducción del riesgo de desastre (RRD), siendo adoptado, sobre todo, después del decenio internacional para la reducción de desastres de la $\mathrm{ONU}^{1}$, tanto en protocolos internacionales como nacionales (Bocco, 2019; Kendra, Clay y Gill, 2018; Aldunce Beilin, Handmer y Howden 2014). Para algunos autores esta noción se ha vuelto el nuevo paradigma de la gestión en RRD (Manyena, 2006), mientras que para otros, desde una perspectiva crítica, lo conciben como un nuevo dispositivo de biopoder gubernamental (De La Fabián y Sepúlveda, 2018; Evans y Reid, 2016) el cual viene a desplazar la causalidad procesual-estructural de la vulnerabilidad social (Macías, 2015) por medio de la reconfiguración ecoterritorial del componente sostenible del desarrollo (y sus estilos de vida inmanentes) promovidos por el «capitalismo de desastres» (Klein, 2011; Fernández y Ruiz-Godoy, 2017). Esta reconfiguración discursiva del desarrollo sostenible, buscaría desmantelar las estructuras públicas de la seguridad social ante riesgos socioambientales, desplazando la responsabilidad hacia las propias comunidades expuestas-susceptibles (ahora comprendidas como empresarias de su adaptación ante el cambio 
ambiental global), ampliando aún más el poder simbólico neoliberal del capitaloceno a nivel subjetivo (Moore, 2016; Han, 2014).

En función de esto, el propósito del presente artículo es doble, en primer lugar, se explora el papel discursivo que juegan las nociones de vulnerabilidad social y resiliencia en la construcción del campo académico-institucional de la RRD; Para, en segundo lugar, aplicar algunas de estas acepciones sobre la producción de vulnerabilidad-resiliencia en una comunidad afectada por un desastre climatológico en Chile. Para esto, se utilizará el marco analítico de la ecología política, esto con el objetivo de ir más allá de los discursos físiconaturalistas como de los progresistas-desarrollistas, esto sin negar la dimensión normativa de la injusticia espacial-ambiental (y su praxis consecuente) subyacente al proceso de riesgo-desastre; así como también, para incitar procesos de (re)apropiación semántico-ontológicos de la noción de resiliencia, hoy categoría hegemónica medular para comprender el campo de acción de la RRD (Laclau, 2005, Leff, 2019; Bocco, 2019).

Para esto, y de acuerdo con Bustos, Prieto y Barton (2015), considero necesario aclarar el uso de las categorías de construcción y producción en la presente investigación. La primera busca enfatizar los procesos discursivos que construyen los sentidos relacionales entre vulnerabilidad-resiliencia (y la materialidad institucional de sus prácticas discursivas); Mientras que el segundo releva el proceso mediante el cual, el capitaloceno extractivista genera un «tipo» de vulnerabilidad-resiliencia glocal, del cual se derivan relaciones socionaturales locales específicas.

\section{Ecología política de los desastres}

La relación entre el proceso de riesgo-desastre y ecología política (EP) es vasto. De acuerdo con RomeroToledo y Romero (2015) este campo se ha nutrido de tres grandes corrientes de la EP: i) Economía política y geografía critica, de la mano de Blaikie y Brookfield (1987), la cual centra su análisis sobre el control y acceso a recursos, bajo un contexto de vulnerabilidad socio-política-económica; ii) una segunda corriente, de tintes posestructuralistas (Escobar, 2007), centrando su interés en las prácticas discursivas y pluriversos de resistencia que dan voz a los «vulnerables-vulnerados», desde sus propios lugares de ser-estar, y; c) la vertiente del actor-red y el post-humanismo, la cual busca difuminar la frontera ilusoria entre cultura y naturaleza, dotando a los peligros naturales y tecnologías el rol de actantes, esto bajo el alero del Antropoceno como concepto cultural-geológico clave (Latour, 2014).

En ALC, el estudio social del proceso de riesgo-desastre presenta una data reciente (Lavell, 2004). Este campo ha sido abordado, con mayor influencia por la $\mathbf{R E D}^{2}$, quienes la han problematizado desde la economía política y geografía critica principalmente, compartiendo algunas categorías analíticas con la EP anglosajona, tales como la i) construcción de lo «natural», ii) producción de conocimiento, iii) poder y iv) derechos de propiedad (Bustos et al. 2015); No obstante, a pesar de estas similitudes con las geopolíticas del conocimiento convencionales, esta propuesta ha sido considerada como una variante política-intelectual independiente y con categorías de análisis propias (Bocco, 2019; Alimonda, 2016). Las contribuciones de la RED han posibilitado nuevas formas de comprender el proceso de riesgo-desastre y su gestión, las cuales se sintetizan en cinco aspectos centrales: i) Los desastres no son naturales, sino construidos socialmente; ii) el nexo inherente entre el riesgo de desastres, modelo de desarrollo y medioambiente; iii) la importancia de los pequeños desastres, así como los riesgos extensivos e intensivos; iv) la importancia de la gobernanza local; y v) la investigación forense del riesgo (Alcántara-Ayala, 2019; García-Acosta, 2005). No obstante, sin negar el aporte político-normativo de este enfoque, este ha tendido a sostener una perspectiva estructuralista del proceso, lo cual ha tendido a instituir una mirada abstracta, mecánica, pasiva y homogénea de las comunidades afectadas (Fernández, Waldmüller y Vega, 2020; Aguirre, 2004).

En esta misma línea, los intentos explícitos en vincular EP y desastres en ALC son escasos. Tres interesantes trabajos son los de Campos-Vargas, Toscana-Aparicio y Campos (2015), Romero-Toledo y Romero (2015) y Sandoval, Boano, González-Muzzio y Albornoz (2015), quienes, desde el abordaje estructural de la primera corriente, destacan el carácter histórico-construido del proceso de riesgo-desastre, identificando los problemas no resueltos del Desarrollo y la vulnerabilidad social como dimensiones explicativas de este. No obstante, si 
bien estas propuestas presentan un carácter pionero al analizar el proceso desde la justicia ambiental, su énfasis tiende a ser macroanalítico y anclada en la categoría de Desarrollo humano y social en abstracto, subordinando el papel del agenciamiento y su variopinta expresión local (Fernández et al. 2020; Sandoval y Astudillo 2019). Por lo tanto, sin negar la influencia de las dimensiones estructurales/procesuales de la vulnerabilidad sobre el proceso de riesgo-desastre, se requiere de una perspectiva dialéctica diferencial, que no sólo dote de historicidad a la exposición-susceptibilidad, sino también, que incorpore los sentidos y practicas sociales multiescalares de afrontamiento, en términos de posiciones agenciales e interseccionales ${ }^{3}$ desde sus particulares pluriversos ecoterritoriales de acción (Escobar, 2007). Desde la literatura especializada, estas capacidades de afrontamiento han tendido a ser conceptualizadas bajo la noción de resiliencia, la cual será analizada, previa inducción a las categorías de a) riesgo-desastre y b) vulnerabilidad.

\section{El proceso de riesgo-desastre}

El concepto de desastre ha sido ampliamente discutido, reflejando distintos antagonismos paradigmáticos (Maskrey, 2011). El paradigma fisicalista concibe los desastres como el choque de amenazas externas, enfatizando tanto las perdidas físicas como la importancia de la respuesta tecnológica-ingenieril ante la crisis (Fernández et al. 2020). La segunda perspectiva, de corte constructivista, enfatiza los orígenes socioestructurales de estos, por medio de las categorías de vulnerabilidad social y/o resiliencia (Tierney, 2014; Wisner et al. 2004).

Por otro lado, el concepto de riesgo se define como el resultado de la interacción de un peligro y la vulnerabilidad del sistema, incluida la probabilidad de que ocurran eventos desastrosos (Birkmann, 2013). Mientras que el riesgo es un continuo, el desastre se entiende como una materialización de este; No obstante, el riesgo no sería sólo un producto de análisis racional de «expertos», sino también una experiencia perceptiva configurada históricamente por materializaciones, artefactos, normas y valores socioculturales de aceptación intersubjetiva (García-Acosta, 2005; Douglas, 1996).

Por tanto, una comprensión profunda y en cámara lenta del proceso de riesgo-desastre (Ultramari y Razende, 2007) requiere, en primer lugar, desplazar la atención desde los peligros hacia la vulnerabilidad diferencial, en la cual tanto susceptibilidad como resiliencia (en su acepción transformadora) formen parte de una misma condición interdependiente (Macías, 2015). De esta forma, los ensamblajes socionaturales no podrían ser analizados sin considerar el entramado de relaciones agenciales territorializadas con el entorno objetivado «paisajísticamente» (ej. los peligros naturales) en términos de algo estático y pasivo, sino como actantes en movimiento que afectan y co-determinan las (in)acciones de afrontamiento (Romero-Toledo y Romero, 2015).

\section{Vulnerabilidad y resiliencia}

Actualmente, el proceso de riesgo-desastre tiende a explicarse con las nociones de vulnerabilidad, adaptación y resiliencia, bajo variados significados, énfasis y configuraciones relacionales (Bocco, 2019). En el campo de RRD, la vulnerabilidad ha tendido a ser conceptualizada en términos espaciales, utilizándose con distintos énfasis: a) para describir el potencial de daño de un sistema físico, b) fragilidad del entorno construido y c) susceptibilidad de perdida de los sistemas socioeconómicos (Cutter, 2013). Desde una perspectiva agencial, Tierney (2014) lo utiliza para denotar niveles de susceptibilidad diferenciada de grupos sociales ante peligros, al igual que Wisner et al. (2004), quienes enfatizan sus capacidades para responder y recuperarse. Es así como este enfoque alternativo al fisicalista, posibilitó la inicial incorporación e explicación de lo «social» sobre el proceso de riesgo-desastre; Sin embargo, su pluralidad disciplinar y conceptual, no sólo ha dificultado el consenso académico, sino también su aplicación institucional en RRD, en términos de operacionalización, medición, evaluación e intervención (Fernández et al. 2020).

Por otro lado, desde fines de los 90 del siglo pasado, el discurso de la resiliencia se ha instalado en una amplia gama de disciplinas e instituciones de RRD, esto bajo una justificación «adaptativa y afirmativa» de la respuesta y recuperación ante desastres (Kendra et al. 2018; Macías, 2015). De acuerdo con la UNDRR, la resiliencia se concibe como las capacidades de un sistema expuesto a una amenaza de origen natural, con el objetivo de anticiparse, resistir, absorber, adaptarse y recuperarse de sus efectos de manera eficaz, para lograr la 
preservación, restauración y mejoramiento de sus estructuras, funciones básicas e identidad (2015a). Sin embargo, esta noción, al igual que la vulnerabilidad, no ha estado exenta de críticas, tanto por sus supuestos, definición, sustancia y aplicación (Bocco, 2019; Aldunce, et al. 2014; Manyena, 2006).

Respecto a su origen y área de aplicación, Norris et al. (2008) engloban los discursos de la resiliencia bajo tres dispositivos disciplinarios:

a. Ciencias físicas e ingeniería: Capacidad de resistencia material para volver a su forma original, o la velocidad con la que se regresa al equilibrio después de un desplazamiento.

b. Ciencias ecológicas: Capacidad de un sistema para absorber una perturbación sin cambiar su estructura original. Esta idea de absorción se convirtió en la capacidad de adaptación que los sistemas complejos describen como autoorganización.

c. Ciencias del comportamiento: Capacidad de individuos y comunidades para resistir, recuperarse y regresar a la línea de funcionamiento basal post impacto de un estresor externo. Es decir, modelos comprehensivos de adaptación positiva manifestado en el contexto de experiencias adversas.

En el campo de riesgo-desastre, Atallah Bacigalupe y Repetto (2019) identifican tres «olas» investigativas sobre resiliencia. La primera denominada i) «rebotar hacia atrás», tiene como objetivo explicar cómo los sistemas expuestos retornan a su estatus quo, centrándose en la respuesta, manejo de la amenaza y normalización funcional. Esta primera ola se sostiene en la meta-narrativa mecanicista-tecnocrática de la adaptación tecnológica (Aldunce et al. 2014), la cual se enfoca en la alerta y respuesta, por medio de sistemas de evaluación de expertos en amenazas naturales y desarrollo de infraestructuras de mitigación. Este ha sido el enfoque hegemónico institucional en RRD, legitimado bajo discursos expertos-ingenieriles y de gestión verticalista del peligro.

La segunda ola, denominada ii) «rebotar hacia adelante», releva los procesos dinámicos de adaptación y reorganización estructural de los sistemas socio-ecológicos, esto ante un entorno complejo de perturbaciones inciertas ante el cambio climático (Adger, 2003). Esta segunda ola, se sostiene en la meta-narrativa de la sostenibilidad (Aldunce et al. 2014), concibiendo la adaptación al cambio ambiental, en términos de «ajuste» entre naturaleza y sociedad, como sistemas complejos interrelacionados. Esta perspectiva establece un análogo entre resiliencia ecológica y resiliencia social (sin dejar muy clara las diferencias entre ambas), relevando su retroalimentación y autoorganización. A su vez, es el actual enfoque hegemónico en lo académico, bajo los aportes de Neil Adger, la Resilience Alliance ${ }^{4}$ y la economía ecológica ortodoxa (Bacco, 2019).

Por último, la tercera ola iii) «centrada en los márgenes», releva el rol agentivo de las comunidades expuestas-susceptibles ante el proceso de riesgo-desastre. Esta última perspectiva se sostiene en la metanarrativa de las capacidades locales (Aldunce et al. 2014), presentando una incipiente cabida en las actuales estrategias internacionales en RRD. Esta perspectiva enfatiza el papel agentivo, formas de organización horizontales, disposiciones adaptativas territoriales y capitales tales como i) conocimiento, experiencia y memoria colectiva (López y Limón, 2017), ii) percepción y aceptación publica del riesgo (García-Acosta, 2005; Douglas, 1996), iii) improvisación y empoderamiento (Kendra et al. 2018) y (iv) capital social, considerando este último como un factor determinante, al permitir vincular practicas locales con desempeño institucional efectivp (Adger, 2003).

Sin embargo, esta noción no ha estado exento de críticas. Macías (2015) sospecha de los enfoques «resilientistas», dado i) el mero relevo comunitario, en desmedro del papel condicionante de las estructuras transescalares, ii) énfasis en la adaptación social, minimizando el papel de la reducción de la vulnerabilidad y una iii) acepción neodarwinista de la adaptación, entendida como mero ajuste al peligro. Por tanto, esta conceptualización permitiría desplazar aquellas problemáticas no resueltas por el Desarrollo, hacia una lectura localista afirmativa, remitiendo la reducción de daño a meros arreglos ingenieriles-tecnológicos, una educación ambiental basada en expertos y el acceso a seguros privados (Aguirre, 2004). Esta lectura resilientista, en clave de «comunidad terapéutica», ha tendido no sólo a soslayar las características procesuales y contextuales de la vulnerabilidad, sino también, a una domesticación de la noción de resistencia, concebida tradicionalmente como un significante con una fuerte impronta contestataria hacia las relaciones de poder, en este caso, hacia el señalamiento de las fuerzas (re)productoras de la vulnerabilidad (Sandoval y Astudillo, 2019). 
Sumado a lo señalado, Bacco (2019) apunta su crítica hacia el enfoque socio-ecológico de la resiliencia (hegemónica en la academia), identificando su i) predominante carácter funcional-estructural, soslayando el rol de la agencia, ii) deshistoricidad de los procesos, iii) ausente conceptualización del poder, iv) comprensión de la adaptación como ajuste, no como transformación, v) propensión al imperialismo científico, en términos de una gran teoría abstraída del contexto, y vi) omisión de la dimensión territorial.

No obstante, si bien compartimos ambas críticas hacia el «resilientismo a secas», consideramos necesaria la (re)apropiación y politización conceptual de este significante flotante (Laclau, 2005), tanto por su actual difusión y sentido práctico en RRD, como por el relevo de la praxis agencial y de componentes adaptativos no sólo de ajuste, sino de potencial transformación territorial bajo la era del capitaloceno (Sandoval y Astudillo, 2019). Por tanto, una primera exploración conlleva, necesariamente, la deconstrucción de las visiones hegemónicas de corte mecánica-tecnocrática en lo institucional y socio-ecológica en la academia, esto hacia una praxis de una resiliencia «centrada en los márgenes» y en el territorio (Atallah et al. 2019); Definiéndolo, tentativamente, como un proceso dialectico transformador de las componentes socionaturales y simbólicos de la vulnerabilidad, ante la cual, la resistencia al poder (subdimensión normativa de la resiliencia) juega un rol central en la (re)configuración de los riesgos, esto bajo el telón de fondo de los sentidos y prácticas de las formas de vida que allí habitan (Escobar, 2007). En consonancia con lo anterior, a continuación, se analiza un caso de estudio a la luz de la proto-propuesta enunciada, asumiendo de antemano su carácter exploratorio y las limitaciones que esta contiene.

\section{Características del caso: El desastre hidrometereológico en el lugar más árido del planeta}

Un evento hidrometeorológico extremo, entre el 24 y 26 de marzo del 2015 (25M), producto de una baja segregada asociada precipitaciones, causo lluvias torrenciales en 17 quebradas en Atacama, Chile. En Copiapó, capital regional, las lluvias arrastraron gran cantidad de agua, piedras, basura y depósitos mineros, enterrando la ciudad bajo una capa de sedimentos de $31 \mathrm{~cm}$ de espesor, dejando a más del 50\% de las viviendas sin sistema de alcantarillado. En cuanto a la afectación, se registraron 22 personas fallecidas, 28.000 damnificados, 2.000 viviendas destruidas y 5.000 con daño mayor, declarándose zona de catástrofe y de estado de excepción constitucional $^{5}$, conllevando un impacto económico superior a 46 millones de USD ${ }^{6}$ (Izquierdo, Abad, Justo, Bernárdez y Arancibia, 2018).

La altura media de afectación fue de $45 \mathrm{~cm}$, identificándose cuatro sectores de la ciudad en los que la inundación superó el metro de lámina de agua, siendo uno de ellos la localidad de Paipote, zona cero del desastre. Dos años después, fuertes lluvias volvieron afectar la región, resultando 221 viviendas destruidas, 3.885 con daños, 5.154 personas afectadas y 204 albergadas.

\section{Materiales y métodos}

En términos metodológicos, este trabajo recoge y articula elementos de investigación acción participativa (IAP). Para esto, se utilizó un diseño de estudio de caso seleccionando a la localidad de Paipote, como un caso representativo, al ser la zona cero del desastre 25M (Gerring, 2007).

Fundamental a este diseño es la fase de producción de información, vinculada a la experiencia participativa directa del investigador a lo largo del ciclo del desastre, alternando instancias de colaboración con agrupaciones vecinales, participación en asambleas, organización de actividades conjuntas, participación en campañas de ayuda a los damnificados, entre otras. Por último, con el fin de incorporar criterios de validación cruzada se incorporó la triangulación intra método (Given, 2008) al utilizar distintas técnicas cualitativas de datos, tanto primarias como entrevistas, observación participante y asistencia a reuniones organizativas, como fuentes secundarias, principalmente informes institucionales y prensa escrita. 


\section{Resultados \\ Producción de la vulnerabilidad en Atacama}

En Atacama existe un histórico desarrollo geográfico desigual en cuanto a la exposición-susceptibilidad ante situaciones de riesgo-desastre, lo cual está lejos de ser causado por el mero peligro o la incierta voluntad divina-natural (Maskrey, 2011). La cronología de los desastres regionales se retrotrae a una larga historia de injusticias espaciales a distintas escalas y niveles, ante las cuales, el modelo de desarrollo extractivista minero ha jugado un rol central en la intensificación de vulnerabilidades basales y de (re)producción de vastos riesgos socioambientales (Gudynas, 2019). Entendemos extractivismo, como las actividades productivas de desposesión territorial que remueven grandes volúmenes de bienes comunes, afectando gravemente a poblaciones históricamente desposeídas y postergadas del progreso desarrollista (Alimonda, 2016). En Atacama, esta producción espacial de zonas de sacrificio en pos del crecimiento nacional, se arrastra desde la época colonial, se intensifica con la acción postcolonial del Estado, y se radicaliza bajo el neoliberalismo (Romero-Toledo, 2019), conllevando una creciente e intensiva apertura de grandes explotaciones mineras vía inversión transnacional (Astudillo, 2014).

Para el despliegue de la minería regional ha sido fundamental la producción de territorios hidrosociales (Swyngedouw, 2015), lo cual ha intensificado la crisis hídrica regional, consumiendo el 80\% del acuífero, junto con la agroindustria (Astudillo, 2014). Completando el panorama del ciclo productivo, en términos de excreciones ambientales, de acuerdo con relaves.org (s.f) Atacama es la segunda ciudad de Chile con mayor cantidad de depósitos de relaves ${ }^{7}$ (161 en total con 30 activos, 108 inactivos y 23 abandonados) constituyendo una fuente de peligro para la salud ambiental, la cual se vio intensificada por la remoción de estos a causa de la inundación del 25M (Corteìs y Tchernitchin, 2018).

Esta producción material de Atacama como territorio extractivista, se ha visto acompañada históricamente por una construcción discursiva legitimadora de la vocación minera regional, pasando del salitre al cobre y en la actualidad al litio (Romero-Toledo, 2019); a lo cual se suma, una elevada dependencia socioeconómica de los trabajadores del rubro (y sus familias), quienes perciben y gestionan, en mayor medida, el riesgo a recibir menores remuneraciones y/o desempleo, por sobre los riesgos ambientales mencionados (Douglas, 1996).

Respecto a la susceptibilidad, entendida como la medida en que una unidad de exposición es afectada en base a sus características demográficas (Cutter, 2013), Atacama presenta cualidades particulares de vulnerabilidad social. Si bien la pobreza por ingresos de la región $(7,9 \%)$ se encuentra por debajo de la media de Chile (8,6\%), esto no se corresponde con la pobreza multidimensional $(23,2 \%)$ la cual se encuentra por sobre la media nacional (20,7\%), ubicándose en el quinto lugar regional al incorporar las dimensiones de i) trabajo y seguridad social, ii) educación, iii) vivienda y entorno, iv) salud y v) redes y cohesión social (CASEN $\left.{ }^{8}, 2018\right)$, condiciones que incrementan la susceptibilidad poblacional ante desastres (Wisner et al. 2004).

En términos de exposición, entendida como el grado espaciotemporal en el cual un grupo podría verse afectado por un peligro (Birkmann, 2013), la ciudad se localiza entre la confluencia de la quebrada Paipote y el río Copiapó. Debido a su localización geomorfológica, la zona ha experimentado a lo menos 11 inundaciones documentadas previas al 25M (Izquierdo et al. 2018), lo cual relevan los procesos, y no la contingencia o «excepcionalidad» en la periodicidad de estos eventos extremos en el lugar más árido del mundo (Vargas y Ortega, 2018).

A partir de estos antecedentes configurantes de las estructuras de vulnerabilidad bajo el capitaloceno, y su consecuente injusticia ambiental y espacial en Atacama, el desastre climatológico emerge como una causa inmanente a los conflictos latentes del extractivismo neoliberal chileno (Moore, 2016: Tierney, 2015). No obstante, para Radcliffe (2005) el neoliberalismo no es un proyecto monolítico de explotación y dominación sin escape, sino un diseño en continua negociación performativa de subjetividades y espacios relacionales (cotidianos e institucionales), desde los cuales es necesario explorar el factor resistente-resiliente a escala local y a nivel comunitario, seleccionando para el presente caso, la localidad de Paipote. 


\section{La localidad $^{9}$ de Paipote}

De acuerdo con Izquierdo et al. (2018), las inundaciones causadas por flujos de derrubios y fango constituyen un riesgo a escala global, especialmente en zonas de grandes desniveles y clima árido, como es el caso de Paipote (Ver figura 1). Esta localidad residencial de 20.000 habitantes, fundada en 1913 bajo el auge ferroviario de la minería regional, se ubica entre la conurbación de las comunas de Tierra Amarilla y Copiapó, a 8 kilómetros de esta última. En términos políticos-administrativos, esta localidad arrastra una larga lucha para convertirse en comuna independiente, para así dejar de ser el «patio trasero» de la capital regional. Esta «sentida» marginación se vincula a la escasa accesibilidad y conectividad a servicios básicos, tanto públicos como privados.

Desde siempre hemos sido el patio trasero de Copiapó, esto independiente de los alcaldes y los partidos políticos ... todos son iguales (Paipotina, 55 años)

En materia educacional, no tenemos liceo y para los servicios básicos tenemos sólo un Servipag ${ }^{10}$, una farmacia y un supermercado. Ni cajero automático tenemos ... para todo tenemos que ir a Copiapó, con las incomodidades y gastos que esto tiene (Paipotina, 60 años)

En cuanto a la cronología del desastre, el 25M se inicia con la escorrentía de lluvia en la quebrada Paipote, la cual se vio desbordada por el estrangulamiento al cauce por un puente ferroviario en desuso (Izquierdo et al. 2018). Es así como este puente, vestigio de la historia minera regional y abandonado desde 1975, se convirtió en un actante clave del desborde hacia zonas residenciales (Latour, 2014).

El puente hizo una especie de topón del aluvión, haciendo que este se desparramase hasta lugares que no hubiesen sufrido daño ... yo creo que, si no hubiese estado ese puente, las cosas no habrían sido tan graves (Paipotino, 65 años)

A su vez, la quebrada se encontraba canalizada por una obra de mitigación ante inundaciones denominada, por la propia comunidad como la defensa. No obstante, la ausencia de eventos hidrometeorológicos, en al menos una década, gatilló que la comunidad expuesta percibiese espacios de relativa seguridad y de inmunidad subjetiva ante este riesgo (Douglas, 1996), manifestado en el escaso conocimiento y preparación (tanto en la utilización de la quebrada como basural, como en la ausencia de planes de acción familiar). Esto último, a pesar de la latente memoria colectiva de inundaciones pasadas por parte de la población adulta mayor (López y Limón, 2017).

Se hizo esta obra [La defensa] por qué que antes de construirla se desbordaba la quebrada; y construyeron una defensa para proteger a Paipote de los desbordes y el nombre se le dio casi solo. La gente la comenzó a llamar la defensa (Paipotino, 70 años)

Escuchamos siempre de nuestros/as papás, lo que les habían contado sus abuelos/as, respecto de las bajadas de la defensa, que hace como 80 años arrasaron con todo ... nunca nos dio miedo, sólo cuando llovía poco o mucho estábamos alerta, porque la defensa estaba a pasos, metros, al frente de nuestras viviendas ... No obstante, la quebrada de Paipote es mucho más, no sólo la canalización que llamamos defensa (Manquez, 2016 p.12-13).

La frase «la defensa no nos defendió» circulaba entre las narrativas de los vulnerados por el desastre, sintetizando así la crítica implícita hacia la inefectividad ingenieril, tanto por su diseño, como por su nulo mantenimiento gubernamental. A partir de esto, paulatinamente los factores causales sociopolíticos de la vulnerabilidad fueron adquiriendo sentido explicativo para los damnificados, al concienciar y desnaturalizar la responsabilidad de la «inusitada» amenaza climatológica, apuntando más bien hacia el (des)ordenamiento y abandono territorial previo. Por otro lado, a nivel gubernamental, predominaron discursos responsabilizantes de tipo fisicalistas-naturalistas, vinculados al cambio climático, reduciendo el rol de las autoridades a la mera respuesta remedial y rehabilitación física-material (Aldunce at al.2014). Por tanto, a nivel comunitario, esta búsqueda de explicaciones sobre las causas humanas sobre el desastre, conllevo la emergencia de nuevos sentidos ontológicos 
sobre lo socionatural del evento, como de improvisados despliegues de afrontamiento resistentes-resilientes ante sus consecuencias y «responsables» (Leff, 2019; Escobar, 2007; Kendra et al. 2018).

Acá no fue el rio el que nos quitó nuestras casas, sino la ausencia del gobierno respecto a la limpieza del cauce y la falta de supervisión de la canalización (Paipotino, 45 años).

Hay que tener claridad con esto, cuando se empieza a buscar responsables. Yo creo que ni los meteorólogos lo sabían. En Copiapó llovió poco, 20 a 30 mm, que es poco, pero no es el agua que inundó la ciudad. Fue la quebrada de Paipote, el río Copiapó, todo lo que viene de las montañas traía agua y agua (Ex alcalde de Copiapó, La Tercera, 2015).

Otro elemento paisajístico de esta zona de sacrificio extractivista, se vincula a la planta fundidora «Hernán Videla» perteneciente a la ENAMI ${ }^{11}$, la cual en 1993 fue declarada zona saturada por anhídrido sulfuro$\mathrm{so}^{12}$ (Instituto Nacional de Derechos Humanos [INDH], 2015). Esta injusticia ambiental basal, se vio acrecentada tras el 25M, dado el desplazamiento aluvional de depósitos mineros (abandonados y activos), exponiendo aún más a la población a elevadas concentraciones de cobre, arsénico y plomo (Corteìs y Tchernitchin, 2018). Esto da cuenta de la paupérrima norma de regulación (y supervisión) sobre estos pasivos ambientales, como de la indiferencia gubernamental ante las nocivas consecuencias de estos sobre la salud poblacional (Relaves.org, s/ f; Izquierdo et al, 2018).

Figura 1

Diagrama aéreo de los principales hitos de Paipote

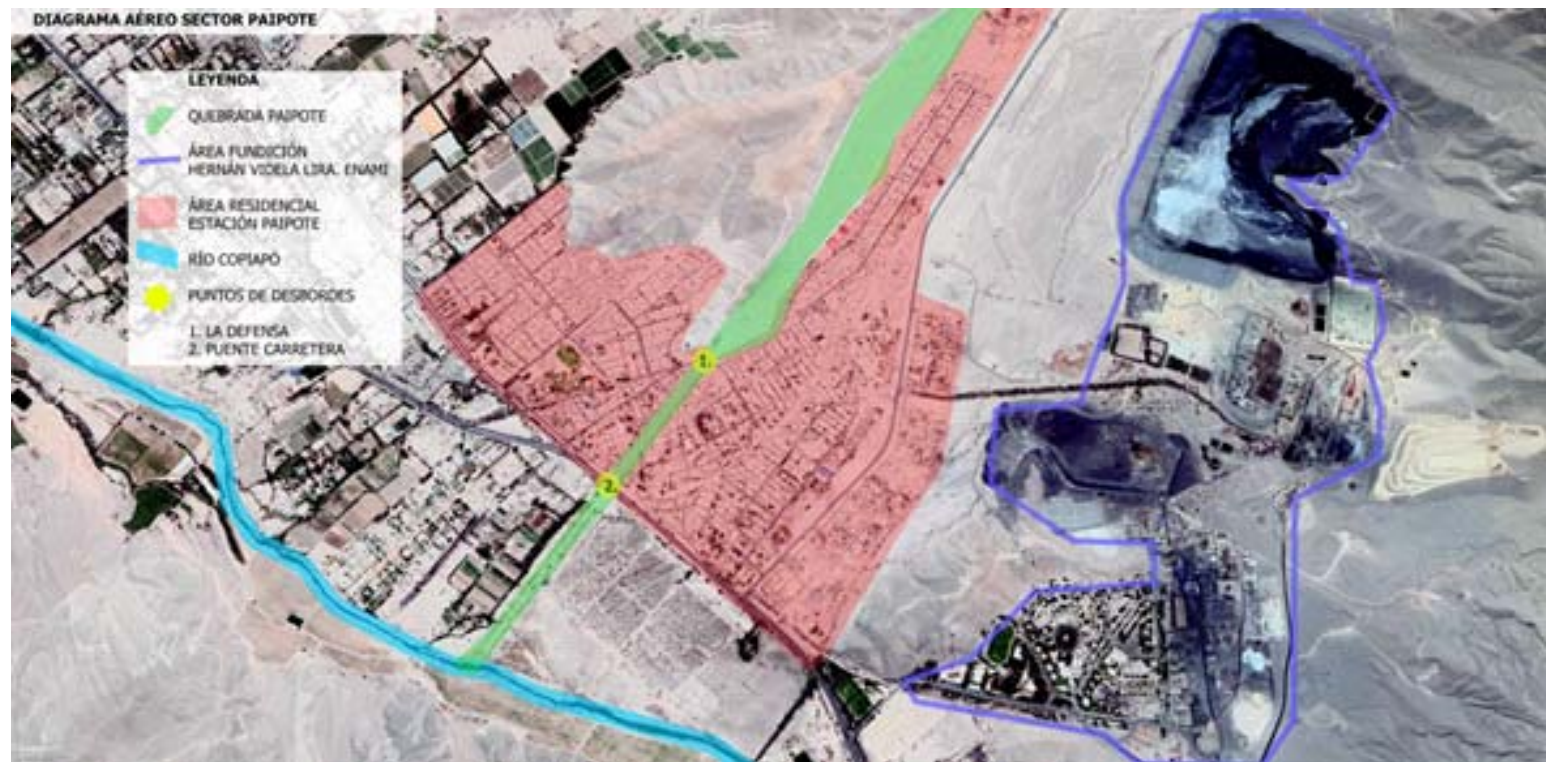

Elaboración propia: Astudillo y Sandoval (2019)

Por tanto, el malestar poblacional pre-desastre, vinculado al desarrollo desigual minero (bajos beneficios económicos, altos costos de contaminación ambiental), se vio intensificado tras los aluviones, posibilitando interpretar el desastre como un problema no resuelto del Desarrollo (Maskrey, 2011). Sin embargo, esta actualización del conflicto distributivo basal post-evento, posibilitó la emergencia de movimientos asambleístas organizados para el afrontamiento (Escobar, 2007), expresándose en prácticas de acción cotidianas, tanto de tipo i) tácticas-resistentes tales como protestas sociales, corte de carreteras y presencia en medios de comunicación ii) como estratégicas-resilientes, bajo movilizaciones colectivas que paralizaron la reubicación habitacional, como en la demanda histórica de una jurisdicción municipal independiente 
de Copiapó, la cual logro su concreción a inicios del año 2019 (Rojas y Sandoval, 2020).

Paipote siempre ha sido mirado como el patio trasero de Copiapó, y por eso apoyamos y creemos que es válida la aspiración que tenemos de concretarse como comuna independiente, ello pues tienen los habitantes necesarios, tienen empresas que pueden aportar permanentemente a su desarrollo integral en conjunto con el gobierno, y tienen su propio movimiento ciudadano que lidera esta iniciativa, y que me gustaría seguir apoyando con todas mis fuerzas. (El Quehay decierto.cl, 2015 p.1)

En la etapa de recuperación pos-desastre, a nivel gubernamental se planteó como medida de solución la expropiación habitacional de la denominada zona cero, esto debido a su alta exposición ante nuevas inundaciones. A nivel nacional, la política de recuperación pos-desastre ha tendido a sostener una lectura basada en la reconstrucción mitigadora de tipo viviendista y cortoplacista, (Contreras y Beltrán, 2015), comprendiendo a los afectados como meros receptores de subsidios individuales, fracturando así el capital social de base de los lugares afectados (Sandoval y Fava, 2016; Rojas y Sandoval, 2020).

A nivel local, dichas decisiones gubernamentales se sancionaron bajo saberes y procedimientos técnicos-legales de corte ingenieril, soslayando el saber popular-territorial de sus propios «habitantes» (Astudillo y Sandoval, 2019). Este saber autorizado del experto o burócrata, cargado de poder decisional, da cuenta de la «distribución desigual de símbolos y legitimidad cultural», dando cuenta de la inconmensurabilidad ontológica y primacía epistémica del conocimiento técnico-científico por sobre el saber encarnado de los habitantes (Leff, 2019; Escobar, 2007). Sin embargo, dicha medida fue resistida por la comunidad organizada y movilizada tanto en el espacio público (plaza local y calle) como en el mundo virtual (Facebook y WhatsApp), que permitieron organizar, deliberar, coordinar y transformar la ira en indignación y está en esperanza colectiva, hasta lograr detener la expropiación (Castells, 2012). Estas prácticas de resistencia ante la expropiación se fundamentaron en la: i) Accesibilidad a servicios sociales y familia extendida, ii) apego al lugar, esto debido a que la mayoría había nacido y crecido allí, y iii) las posibilidades de ser-estar que brindaban las viviendas a expropiar, en contraposición a los potenciales apartamentos minimalistas.

Esta percepción y aceptabilidad diferenciada de los riesgos urbanos, resalta las contradicciones entre la percepción de riesgos propio de los técnicos-expertos, fundada en la exposición y mitigación de amenazas naturales, frente a las comunidades residentes, quienes relevan el derecho a su hábitat y la forma de vida que allí las identifica (Douglas, 1996; Astudillo y Sandoval, 2019). Esta carga de saberes y sentidos normativos diferenciados, entre «lugares» para quienes lo habitan, frente al espacio mensurable de la exposición, para las autoridades, no trata de un problema de inconmensurabilidad sobre la espacialidad del riesgo, sino más bien de identidades-valoraciones diferenciadas que territorializan ese espacio (Leff, 2019);

Para la comunidad de Paipote (cuyo significado vernáculo mapudungun se traduce como «melancolía del desierto») remite a una especie de sentimiento de apego o «topofilia» que apropia e identifica a esta comunidad a su «lugar-de-ser», no recayendo en la reconstrucción topográfica del espacio geométrico de los expertos, sino más bien en los modos topológicos del habitar (Tuan, 2007).

Dos años después del 25M, entre el 10 y 13 de mayo, fuertes lluvias volvieron a inundar Atacama, instancia en la que el plan de reconstrucción sólo presentaba un 40\% de avance (Gobierno Regional de Atacama, 2017). Sin embargo, parte de los hogares previamente afectados, para este momento si contaban con un plan de emergencia familiar, esto a raíz de la experiencia vivida tras el 25M (López y Limón, 2017), así como con seguros y medidas de mitigación habitacionales, basados en recursos entregados por la municipalidad y movilizados por las organizaciones barriales tras la etapa de alerta temprana.

Si bien las asambleas territoriales se replegaron a sus hogares terminada la reconstrucción de las viviendas post 25M, ante este nuevo evento, el capital social comunitario de los barrios se activó y desplegó rápidamente a nivel horizontal (barrios) y vertical (municipalidad), posibilitando una capacidad de respuesta local más rápida y efectiva (Adger, 2003).

Para este aluvión (2017) por lo menos ya teníamos un plan de qué hacer y donde encontrarnos, en este 
caso en la casa de mi mama, no como el del 2015 que nos encontró a todos en distintos lados y sin posibilidad de comunicarnos (se cayeron las redes) ... cuando dieron la alerta preventiva en la radio, nos dividimos las funciones, los niños desconectaban y guardaban las cosas, mientras que con la Juana (esposa) nos dedicábamos a ordenar los sacos afuera de la casa (Paipotino, 40 años)

Del aluvión pasado quedo la organización, yo por ejemplo fui la delegada del pasaje anterior y ahora me toco seguir no más para encargarme de ver el tema de los sacos y los plásticos con la municipalidad ...por medio del whatsapp del pasaje nos preguntábamos que nos faltaba y en que podíamos ayudar (Paipotina, 55 años)

En cuanto a la recuperación pos-desastre, a raíz de la cancelación de la expropiación habitacional a manos de las organizaciones de resistencia, el Ministerio de Obras Públicas (MOP) desarrolló una serie de intervenciones mitigadoras, con el objetivo de dar mayor capacidad a la defensa, ampliándola de 12 a 25 metros (Gobierno Regional de Atacama, 2017). No obstante, a la fecha de término del presente escrito, dichas medidas de mitigación no se han visto acompañada de medidas institucionales de reducción de la vulnerabilidad social, en términos de mayor conectividad, servicios y eliminación de pasivos ambientales, ni tampoco de fortalecimiento de capacidades de afrontamiento comunitarias.

\section{Discusión}

El presente artículo tuvo como objetivo analizar la construcción y producción de la vulnerabilidadresiliencia en el campo académico-institucional de riesgo-desastre socionatural, esto bajo el estudio de un caso climatológico. Para esto utilizamos el marco analítico de la ecología política, esto con el objetivo de desnaturalizar el esencialismo fisicalista-naturalista de los desastres y relevar la ecologización de las relaciones sociales y de poder que se dan al interior de este proceso (Leff, 2019). A modo de conclusión relevamos el uso de enfoques exploratorios comprehensivos, dialecticos y territorializados del proceso de riesgo-desastre socionatural, esto bajo el establecimiento de diálogos transescalares y de multinivel entre la dimensión estructutrante de la vulnerabilidad social (Wisner et al. 2004; Cutter, 2013) y los componentes agentivos-actantes de las formas de vida que configuran los lugares de riesgos, tanto en termino de resistencia ante las injusticias basales, como de resiliencia ante la transformación de estos entornos glocales (Sandoval y Astudillo, 2019).

En primer lugar, la construcción discursiva de la vulnerabilidad-resiliencia ha tendido a ser variada y polisémica, no sólo en términos académicos-disciplinares sino también desde el contexto aplicado de la RRD. De acuerdo con lo revisado, a nivel institucional se tiende a reducir la vulnerabilidad a la mera exposición y/o susceptibilidad física, concibiendo la resiliencia como una respuesta mitigadora de tipo mecánica-tecnológica ante el peligro. Por otro lado, a nivel académico, es hegemónica la postura sistémica socio ecológica de la vulnerabilidad-resiliencia, esto bajo una lectura dinámica pero apolítica de los procesos, concibiendo la adaptación como mero ajuste ante los peligros (Bacco, 2019; Macias, 2015). Ambas perspectivas reducen la adaptación a meros factores funcionales, lineales, abstractos, descontextualizados y acríticos de las relaciones estructurantes de poder subyacentes. Por tanto, se torna necesaria una «adaptación con tintes transformadores» que no sólo fortalezca las capacidades locales de afrontamiento en términos de ajuste, sino también que sea acompañada de reducción de la vulnerabilidad socioterritorial (Sandoval y Astudillo, 2019).

En contraposición a estas perspectivas «resilientistas» y en línea con las perspectivas criticas mencionadas (Bacco, 2019; Macias, 2015; Tierney, 2015), proponemos una (re)apropiación y politización de una resiliencia «centrada en los márgenes» desde los territorios estudiados (Atallah et al. 2019), donde la resistencia ante la (re)producción de injusticias espaciales/ambientales juegue un rol normativo central. Por tanto, la resiliencia, más que una categoría analítica, es una praxis transformadora de los patrones (re)productores del capitaloceno, emergente tras la concienciación de las estructuras de vulnerabilización. Esta praxis de la resiliencia (Atallah et al. 2019) en lo procedimental, se debiera nutrir de una i) ecología de saberes híbridos, tanto de conocimientos vernáculos como expertos, ii) métodos participativos e implicativos desde los propios territorios, relevando la importancia del «lugar», iii) análisis interseccional de la vulnerabilidad social, en términos, de clase, género y etnia y iv) y una gobernanza de los riesgos sustentada en las formas de vida de los territorios (Escobar, 2007). 
En segundo lugar, bajo el Capitaloceno (Moore, 2016), el modo de producción extractivista ha intensificado no sólo las desigualdades basales, sino también el aumento de efectos climatológicos colaterales tales como sequias, incendios forestales e inundaciones (IPCC, 2014). Sin embargo, el factor agencial de resistenciaresiliencia ha sido crucial, para la i) politización y responsabilización de las causas socionaturales de estos desastres ${ }^{13}$ (Astudillo y Sandoval, 2019), ii) emergencia de agentes políticos no tradicionales (Ugarte y Salgado, 2014), iii) fortalecimiento del capital social basado en el apego al lugar (Rojas y Sandoval, 2020), iv) relevo de saberes y memoria territorial (López y Limón, 2017) y v) percepción-aceptación diferenciada de los riesgos (García-Acosta, 2005). Sin embargo, este énfasis en las comunidades como agentes empoderados y activos (Sandoval y Astudillo, 2019), no debe confundirse con la autogestión promovida por el discurso resilientista del «Antropoceno», el cual desplaza el traspaso de la responsabilidad de la sustentabilidad desde el Estado, hacia los propios sujetos, quienes deben hacerse cargo tanto de la aceptabilidad de los riesgos glocales (los cuales muchas veces no son parte ni de sus decisiones ni de su control), como de su proto-construcción como emprendedores de la seguridad (De La Fabián y Sepúlveda, 2018; Evans y Reid, 2016). Por lo tanto, si bien es importante enfatizar el rol activo de las comunidades en la RRD, es importante reconocer que existen límites respecto a lo que pueden lograr las comunidades por siì solas, ya que varios de los factores subyacentes de riesgo son el resultado de procesos estructurales de vulnerabilidad que escapan a las posibilidades del sólo manejo local-territorial (Sandoval et al. 2018; Maskrey, 2011).

\section{Notas}

\footnotetext{
${ }^{1}$ Organización de naciones unidas.

${ }^{2}$ Red de estudios sociales sobre la prevención de desastres.

${ }^{3}$ Estudio de las identidades sociales solapadas, tales como raza, genero, étnica y sus respectivos sistemas de opresión, dominación o discriminación,

${ }^{4}$ Organización de investigación que se enfoca en la resiliencia de los sistemas socio-ecológicos como base para la sostenibilidad. Página web https://www.resalliance.org/.

${ }^{5}$ Mecanismo por el cual se altera la situación regularidad de los derechos y libertades de las personas garantizadas por la constitución política, debido a razones extraordinarias y graves, como lo son los desastres.

${ }^{6}$ Dólar estadounidense.

${ }^{7}$ Conjunto de desechos de procesos mineros de la concentración de minerales, usualmente constituido por una mezcla de rocas molidas, agua y minerales.

${ }^{8}$ Encuesta de caracterización socioeconómica de Chile.

${ }^{9}$ Etimológicamente la noción de localidad es definida como «cualidad perteneciente a un lugar«.

${ }^{10}$ Oficina de pago de múltiples servicios domésticos en Chile.

${ }^{11}$ Empresa nacional de minería.

${ }^{12}$ Gas incoloro, de olor fuerte e irritante, que resulta de la combustión del azufre.

${ }^{13}$ Un ejemplo de esto es la frase «En Chile no hay sequía, sino saqueo» acuñada por el movimiento de defensa por el acceso al agua, la tierra y la protección del medioambiente (MODATIMA)
}

\section{Bibliografía}

Adger, W. (2003). Social capital, collective action, and adaptation to climate change. Economic geography, 79(4), 387-404. doi:10.1111/j.1944-8287. 2003.tb00220.x

Aguirre, B. (2004). Los desastres en Latinoamérica: vulnerabilidad y resistencia. Revista mexicana de sociología, 66(3), 485-510. doi:10.2307/3541400 
Alcántara Ayala, I. (2019). Time in a bottle: challenges to disaster studies in Latin America and the Caribbean. Disasters, 43(1), 18-27. doi:10.1111/disa.12325

Aldunce, P., Beilin, R., Handmer, J., y Howden, M. (2014). Framing disaster resilience: the implications of the diverse conceptualisations of «bouncing back». Disaster Prevention and Management, 23(3), 252-270. doi: 10.1108/DPM-07-2013-0130

Alimonda, H. (2016). Notas sobre la ecología política latinoamericana: Arraigo, herencias y diálogos. Revista de Ecología Política: Cuadernos de Debate Internacional, 51, 36-42. Recuperado de https:// www.ecologiapolitica.info/?p=6017

Astudillo, F. (2014). ¿Acumulación por desposesión hídrica? Crecimiento inmobiliario, neoliberalismo minero y mercantilización del agua en Copiapó, Chile. Revista de Ecología Política: Cuadernos de Debate Internacional, 47, 62-66. Recuperado de https://www.ecologiapolitica.info/?p=1626

Astudillo. F y Sandoval, J. (2019). Justicia espacial, desastres socionaturales y políticas del espacio. Dinámicas sociopolíticas frente a los aluviones y proceso de recuperación en Copiapó, Chile. Cuadernos de Geografía: Revista Colombiana de Geografía, 28 (2), 303-321. doi: 10.15446/rcdg.v28n2.73520

Atallah, D. (2016). Toward a decolonial turn in resilience thinking in multifaceted disasters: Example of the Mapuche from southern Chile on the frontlines and faultlines. International Journal of Disaster Risk Reduction, 19, $92-$ 100. doi:10.1016/j.ijdrr.2016.08.027

Atallah, D., Bacigalupe, G., y Repetto, P. (2019). Centering at the Margins: Critical Community Resilience Praxis. Journal of Humanistic Psychology, 46(1), 48-76. doi:10.1177/0022167818825305

Birkmann J. (2013) Risk. En P. Bobrowsky (Ed), Encyclopedia of Natural Hazards. Encyclopedia of Earth Sciences Series (pp. 856-861). Dordrecht, Holland: Springer.

Blaikie, P. y Brookfield, H. (1987). Land degradation and society. New York, EE.UU: Methuen y Co. Ltd.

Bocco, G. (2019). Vulnerabilidad, adaptación y resiliencia sociales frente al riesgo ambiental. Teorías subyacentes. Investigaciones Geográficas, (100), 1-16. doi:10.14350/rig.60024

Bustos, B; Prieto, M. y Barton, J. (2015). Ecología Política en Chile: naturaleza, propiedad, conocimiento y poder. Santiago, Chile: Editorial Universitaria.

Campos-Vargas, M., Toscana-Aparicio, A, y Campos, J. (2015). Riesgo socionaturales: Vulnerabilidad socioeconómica, justicia ambiental y justicia espacial. Cuadernos de Geografía: Revista Colombiana de Geografía, 24 (2): 53-69. doi:10.15446/rcdg.v24n,2.50207

Encuesta de caracterización socioeconómica (2018). Resultados CASEN 2017. Santiago; Chile: Ministerio de Desarrollo Social de Chile. Recuperado de http://observatorio.ministeriodesarrollosocial.gob.cl/casenmultidimensional/casen/casen_2017.php.

Castells, M. (2012). Redes de indignación y esperanza: Los movimientos sociales en la era de Internet. Madrid, España: Alianza.

Contreras, Y. y Beltrán, M. (2015). Reconstruir con capacidad de resiliencia: El casco histórico de la ciudad de Constitución y el sitio del desastre del terremoto y tsunami del 27 de febrero 2010. Revista INVI, 30(83), 79115. doi:10.4067/S0718-83582015000100003

Corteìs, I. y Tchernitchin, A. (2018). Metales y metaloides en muestras de polvo depositado en diferentes sectores de Atacama, afectados por los aluviones de marzo 2015. En G. Vargas, S. Perez y P. Aldunce (Eds.), Aluviones 
y resiliencia en atacama: construyendo saberes sobre riesgos y desastres (pp. 181-200). Santiago, Chile: Social-Ediciones.

Cutter, S. (2013) Vulnerability. En P. Bobrowsky (Ed), Encyclopedia of Natural Hazards. Encyclopedia of Earth Sciences Series (pp. 1888-1890). Dordrecht, Holland: Springer.

De La Fabián, R. y Sepúlveda, M. (2018). Gubernamentalidad neoliberal postsecuritaria y resiliencia: una nueva metafísica de la identidad. Athenea Digital. Revista de Pensamiento e investigación social, 18(3), 1-26. doi: 10.5565/rev/athenea.211

Douglas, M. (1996). La aceptabilidad del riesgo según las ciencias sociales. Buenos Aires, Argentina: Paidós

El quehay decierto. (25 de diciembre 2015). Piden un regalo de navidad anticipado: ¿Paipote comuna? Recuperado de http://www.elquehaydecierto.cl/noticia/sociedad/piden-un-regalo-de-navidad-anticipado-paipotecomuna\#comments.

Escobar, A. (2007). La invención del tercer mundo: Construcción y deconstrucción del desarrollo. Caracas, Venezuela: Fundación editorial el perro y la rana.

Evans, B. y Reid, J. (2016). Una vida en resiliencia: El arte de vivir en peligro. Ciudad de México, México: Fondo de cultura económica.

Fernández, A., Waldmüller, J. y Vega, C. (2020). Comunidad, vulnerabilidad y reproducción en condiciones de desastre. Abordajes desde América Latina y el Caribe. Íconos. Revista de Ciencias Sociales, 66(24), 7-29. doi:10.17141/iconos.66.2020.4156

Fernández, L. y Ruiz-Godoy, J. (2017). La evolución discursiva de la sostenibilidad a la resiliencia: ¿Un problema ético? Revista de Ecología Política: Cuadernos de Debate Internacional, (53), 34-38. Recuperado de https:/ /www.ecologiapolitica.info/?p=9707

García-Acosta, V. (2005). El riesgo como construcción social y la construcción de riesgos. Desacatos, 19, 11-24. doi:10.29340/19.1042

Gerring, J. (2007). What Is a Case Study and What Is It Good for? American Political Science Review, 98(2), 341354. doi:10.1017/S0003055404001182

Given, L. (2008). The Sage encyclopedia of qualitative research methods. Alberta, Canada: Sage.

Gobierno Regional de Atacama. (31 de mayo 2017). Intendente de Atacama, Miguel Vargas: «Hemos trabajado de manera responsable en el Plan de Reconstrucción 2015 y de esta nueva emergencia nos estamos haciendo cargo». Recuperado de https:/goreatacama.gob.cl/2017/05/intendente-de-atacama-miguel-vargas-hemos-trabajado-de-manera-responsable-en-el-plan-de-reconstruccion-2015-y-de-esta-nueva-emergencia-nos-estamoshaciendo-cargo/

Gudynas, E. (2019). Múltiples paradojas: ciencia, incertidumbre y riesgo en las políticas y gestión ambiental de los extractivismos. Polisemia, 14(25), 5-37. doi:10.26620/uniminuto.polisemia.14.25.2018.5-37

Han, B-C. (2014). Psicopolítica: neoliberalismo y nuevas técnicas de poder. Barcelona, España: Herder Editorial.

Instituto Nacional de Derechos Humanos. (2015). Informe misión de observación a las comunas de Copiapóì, tierra amarilla y ChanParal. Copiapó, Chile: INDH. Recuperado de http://bibliotecadigital.indh.cl/bitstream/ handle/123456789/883/informe-mision-copiapo.pdf?sequence=4.

Intergovernmental Panel on Climate Change. (2014). AR5 Climate Change 2014: Impacts, Adaptation, and 
Vulnerability. New York, EE. UU: Cambridge University Press. Recuperado de https://www.ipcc.ch/report/ ar5/wg2/

Izquierdo, T., Abad, M., Justo, B., Bernárdez., E. y Arancibia, M. (2018). El evento hidrometeorológico del 25M en la ciudad de Copiapó: análisis de la inundación y los daños en el casco urbano. En G. Vargas, S. Perez y P. Aldunce (Eds.), Aluviones y resiliencia en atacama: construyendo saberes sobre riesgos y desastres (pp. 117-135). Santiago, Chile: Social-Ediciones.

Kendra, J., Clay, L. y Gill, K. (2018). Resilience and Disaster. En H. Rodríguez, W. Donner y J. Trainor (Eds.), Handbook of disaster research (pp. 87-108). Cham, Suiza: Springer. doi:10.1007/978-3-31963254-4

Klein, N. (2011). La doctrina del shock: El auge del capitalismo del desastre. Buenos Aires, Argentina: Paidos.

Laclau, E. (2005). La razón populista. Buenos Aires, Argentina: Fondo de cultura económica.

La Tercera. (27 de marzo del 2015). Alcalde de Copiapó revive la fatídica noche cuando inició evacuación de la ciudad tras el desborde del río. Recuperado de https://www.latercera.com/noticia/alcalde-de-copiapo-revivela-fatidica-noche-cuando-inicio-evacuacion-de-la-ciudad-tras-el-desborde-del-rio/

Latour, B. (2014). Agency at the Time of the Anthropocene. New literary history, 45(1), 1-18. doi:10.1353/ nlh.2014.0003

Lavell, A. (2004). Vulnerabilidad social: una contribución a la especificación de la noción y sobre las necesidades de investigación en pro de la reducción del riesgo. En Centro Regional de Información sobre Desastres para América Latina y el Caribe [CRID]. Lima, Perú: INDECI. Recuperado de http://bvpad.indeci.gob.pe/doc/pdf/ esp/doc852/doc852-contenido.pdf

Leff, E. (2019). Ecología Política: De la deconstrucción de capital a la territorialización de la vida. Ciudad de México, México: Siglo XXI editores.

López, F. y Limón, F. (2017). Componentes del proceso de resiliencia comunitaria: conocimientos culturales, capacidades sociales y estrategias organizativas. PSIENCIA. Revista Latinoamericana de Ciencia Psicológica, 9(3) 1-13. doi:10.5872/psiencia/9.3.61

Macías, J. (2015). Crítica de la noción de resiliencia en el campo de estudios de desastres. Revista Geográfica Venezolana, 56(2), 309-325. Recuperado de https://www.redalyc.org/pdf/3477/347743079009.pdf

Manquez, B. (2016). La defensa mi defensa. Testimonio aluvión norte de Chile 2015. Santiago, Chile: Editorial Escritores.cl

Manyena, S. (2006). The concept of resilience revisited. Disasters, 30(4), 434-450. doi:10.1111/j.03613666.2006.00331.x

Maskrey, A. (2011). Revisiting community-based disaster risk management. Environmental Hazards, 10(1), 42-52. doi:10.3763/ehaz.2011.0005

Moore, J. (2016). ¿Anthropocene or Capitalocene? Nature, History, and the Crisis of Capitalism. Oakland, EE.UU : PM Kairos.

Norris, F, Stevens, S, Pfefferbaum, B, Wyche, K. y Pfefferbaum, R. (2008). Community resilience as a metaphor, theory, set of capacities, and strategy for disaster readiness. American journal of community psychology, 41(1), 127-150. doi:10.1007/s10464-007-9156-6 
Obradovich, N, Tingley, D. y Rahwan, I. (2018). Effects of environmental stressors on daily governance. Proceedings of the National Academy of Sciences, 115(35), 8710-8715. doi:10.1073/pnas.1803765115

Oliver-Smith, O., Alcántara-Ayala, I., Burton, I. y Lavell, A. (2016). Investigación forense de desastres: Un marco conceptual y guía para la investigación. Ciudad de México, Mexico: Instituto de Geografía, UNAM. Recuperado de http://www.publicaciones.igg.unam.mx/index.php/ig/catalog/book/71

Pesaresi, M, Ehrlich, D, Kemper, T, Siragusa, A, Florczyk, A, Freire. S. y Corbane, C. (2017). Atlas of the Human Planet 2017: Global Exposure to Natural Hazards. EU: Publications Office of the European Union. doi:10.2760/19837

Radcliffe, S. (2005). Neoliberalism as we know it, but not in conditions of its own choosing: a commentary. Environment and Planning A: Economy and Space. 37(2), 323-329. doi:10.1068/a37407

Relaves. (s.f). ¿Dónde están los relaves? Recuperado de https://www.relaves.org/donde_estan.php

Rojas, L. y Sandoval, J. (2020). Habitando 'no lugares': subjetividad y capacidades familiares ante un desastre socionatural en Chile. Íconos. Revista de Ciencias Sociales, 66(24), 59-80. doi:10.17141/iconos.66.2020.3883

Romero-Toledo, H. (2019). Extractivismo en Chile: la producción del territorio minero y las luchas del pueblo aimara en el Norte Grande. Colombia Internacional, 98(1). 3-30. doi:10.7440/colombiaint98.2019.01

Romero-Toledo, H. y Romero, H. (2015). Ecología política de los desastres: vulnerabilidad, exclusión socio-territorial y erupciones volcánicas en la Patagonia chilena. Magallania (Punta Arenas), 43(3), 7-26. doi:10.4067/ S0718-22442015000300002

Sandoval, J. y Fava, D. (2016). Significados y vulnerabilidad social ante el terremoto y tsunami del 27 de febrero del 2010: la dimensión subjetiva desde los «damnificados». Summa Psicológica, 13(2). 23-32. doi:10.18774/ 448x.2016.13.306

Sandoval., J, Rojas, L., Villalobos, M., Sandoval, C., Moraga, F. y Aguirre, N. (2018). De organización vecinal hacia la gestión local del riesgo: diagnóstico de vulnerabilidad y capacidad. Revista INVI, 33(92), 155-180. doi:10.4067/ S0718-83582018000100155

Sandoval, J. y Astudillo, F. (2019). Comunidades en movimiento ante el cambio climático. ¿Resistentes o resilientes? El caso de Paipote, Chile. Ecología Política: Cuadernos de debate internacional, 58, 79-83. Recuperado de https://www.ecologiapolitica.info/?p=12988

Sandoval, V., Boano, C., González-Muzzio, C. y Albornoz, C. (2015). Explorando potenciales vínculos entre resilencia y justicia ambiental: el caso de Chaitén, Chile. Magallania (Punta Arenas), 43(3), 37-49. doi:10.4067/ S0718-22442015000300004

Swyngedouw, E. (2015). Liquid Power: Contested Hydro-Modernities in Twentieth-Century Spain. Londres, UK: MIT Press.

Tierney, K. (2014). The social roots of risk: Producing disasters, promoting resilience. California, EE.UU: Stanford University Press.

Tierney, K. (2015). Resilience and the Neoliberal Project: Discourses, Critiques, Practices-And Katrina. American Behavioral Scientist, 59(10), 1327-1342. doi:10.1177/0002764215591187

Tuan, Y-F. (2007). Topofília: Un estudio de las percepciones, actitudes y valores medioambientales. Tenerife, España: Melusina. 
Ugarte, A. y Salgado, M. (2014). Sujetos en emergencia: acciones colectivas de resistencia y enfrentamiento del riesgo ante desastres; el caso de Chaitén, Chile. Revista INVI, 29(80), 143-168. doi:10.4067/S071883582014000100006

Ultramari, C. y Rezende, D. (2007). Urban resilience and slow motion disasters. City \& Time, 2(3), 47-64. Recuperado de http://www.ceci-br.org/novo/revista/docs2007/CT-2007-79.pdf

UN Office for Disaster Risk Reduction (2015a). Sendai Framework for Disaster Risk Reduction 2015-2030. Geneva: UNDRR.

(2015b). Marco de Sendai para la Reducción del Riesgo de Desastres 2015 2030. Ginebra, Suiza: UNDRR.

Vargas, G. y Ortega, C. (2018). El Niño y los mayores episodios históricos en Chañaral de las Ánimas, Atacama. En G. Vargas, S. Pérez y P. Aldunce (Eds.), Aluviones y resiliencia en atacama: construyendo saberes sobre riesgos y desastres (pp. 35-94). Santiago, Chile: Social-Ediciones.

Wisner, B., Blaikie, P., Cannon, T., y Davis, I. (2004). At risk: natural hazards, people's vulnerability and disasters ( $2^{\mathrm{a}}$ ed.). London, Uk: Routledge. 